\title{
La influencia de la economía en el voto en España: de la bonanza de 2000 a la crisis de $2011^{1}$
}

\author{
Alberto Mora Rodríguez \\ UNIVERSIDAD DE MURCIA \\ alberto.mora@um.es \\ Cristina Moreno Martínez \\ UNIVERSIDAD DE MURCIA \\ cmoreno@um.es
}

Resumen: Esta investigación pretende contrastar la teoría de la asimetría del voto económico para el caso español. Dicha teoría asume que los efectos del voto económico son mayores en épocas de crisis que en períodos de prosperidad económica. Es decir, se parte de que el castigo en las urnas como consecuencia de situaciones económicas desfavorables, es mayor que el premio en situaciones de bonanza. Las conclusiones de Fraile (2002) refutaron este planteamiento en España, analizando los casos de 1996 y 2000. En este estudio se presenta nueva evidencia, comparando los efectos de la economía en el voto en las elecciones de 2000 y 2011. Para el análisis, se utiliza una ecuación de voto usando la técnica de la regresión logística, con datos de estudios de panel del Centro de Investigaciones Sociológicas. En el modelo elaborado para el análisis, se incluyen indicadores que dan cuenta de los principales debates teóricos que vertebran el estudio del voto económico. Las conclusiones apoyan las del estudio citado, discutiéndose los resultados del análisis a la luz de las especificidades de los comicios analizados.

Palabras clave: voto económico, asimetría del voto económico, comportamiento electoral

Abstract: This research aims to contrast the theory of asymmetry of economic vote in Spain. This theory assumes that the effects of economic vote are greater in times of crisis than in periods of economic prosperity. That is to say, the punishment as a result of unfavorable economic situations is greater than the prize in bonanza situations. The conclusions of Fraile (2002) refuted this approach in Spain, analyzing the cases of 1996 and 2000 Spanish general election. This study offers new evidence, comparing the effects of the economy on the vote in the 2000 and 2011 Spanish general election. We model a vote equation using logistic regression from survey data elaborated by the Centre of Sociological Research. The analysis includes indicators that account for the main theoretical debates on the field of economic vote. The conclusions support those of the cited study, discussing the results of the analysis in the light of the specificities of the analyzed elections.

Keywords: economic vote, asymmetry of economic vote, electoral behavior

1. Esta investigación es una revisión de uno de los análisis incluidos en la tesis doctoral «El voto económico y estudio de agendas en España: aproximación a sus efectos en las elecciones generales del 2000 y 2011» (Mora, 2017). 


\section{Introducción}

as elecciones son un mecanismo inherente a cualquier sistema político democrático, por lo que el estudio del comportamiento electoral es uno de los ámbitos más prolíficos en Ciencia Política. El desarrollo de estas investigaciones ha visto sucederse diferentes debates, uno de los cuales se vertebra en torno al voto económico.

Esta investigación tiene por objeto contrastar la teoría de la asimetría del voto económico (Bloom y Price, 1975) para el caso español. Dicha teoría asume que las magnitudes de los efectos del voto económico son mayores en épocas de crisis que en períodos de prosperidad económica. Es decir, se parte de que el castigo en las urnas como consecuencia de situaciones económicas desfavorables, es mayor que el premio en situaciones de bonanza; hipótesis que ha sido contrastada ${ }^{2}$. Sin embargo, en España Fraile (2002: 145) obtuvo resultados que refutan este planteamiento, al concluir que los efectos del voto económico fueron mayores en España en las elecciones generales de 2000 (bonanza) que en las de 1996. Esta investigación pretende ofrecer nueva evidencia para el caso español, comparando los efectos de la economía en el voto en las elecciones de 2000 y 2011. Dos citas electorales elegidas por varios motivos: Aunque en ambas campañas electorales el eje es la economía; son dos momentos del ciclo económico antagónicos: en el año 2000 el Partido Popular se postula a la reelección tras una legislatura que finaliza con destacados resultados económicos, mientras que en 2011 el Partido Socialista Obrero Español se presenta a las elecciones tras tres años de una importante crisis económica. En las elecciones generales del año 2000 , el PP revalida y amplía su victoria, mientras que el PSOE pierde el poder en las elecciones generales del año 2011.

La selección de los años objeto de estudio resulta clave en el interés de este estudio, pues permite contrastar cómo operan las variables económicas en el voto, en contextos económicos antagónicos, y de esta forma determinar si la magnitud del premio al gobierno es mayor o menor que la del castigo. Sin embargo, es importante matizar algunas limitaciones que supone el análisis comparado de escenarios tan dispares. En primer lugar, al realizar un análisis individual del voto con datos de encuesta, hay que tener en cuenta la escasa muestra que se sitúa en posiciones optimistas respecto a la economía en contextos críticos, lo que limita la capacidad de inferencia de los resultados. En segundo lugar, debe considerarse la posibilidad de que aquellos entrevistados que presentan valoraciones positivas sobre la situación y gestión económica, estén realizando un juicio adaptado a sus afinidades políticas, de manera que no sean las variables económicas las que expliquen el resultado, sino las 
predisposiciones políticas. A pesar de ello, y dado que para el análisis se utilizará una ecuación de voto usando la técnica de la regresión logística, con datos de estudios del CIS, se procurará incorporar las variables imprescindibles para controlar el efecto de la ideología y otros factores intervinientes en la decisión de voto, limitando en la medida de lo posible la pérdida de casos.

Formalmente, este trabajo se estructura en cinco apartados. En el primero se revisan las diferentes escuelas del comportamiento electoral, incidiendo en las teorías del voto económico e introduciendo nuestra principal hipótesis. El segundo da cuenta del contexto económico de los dos procesos electorales objeto de estudio. El tercero se dedica a la metodología. En el cuarto se aporta evidencia empírica sobre el efecto de la economía en el voto, en el año 2000 y en el 2011. Por último, se presentan los principales resultados del estudio, así como una breve discusión.

\section{El estudio del comportamiento electoral y la influencia de la economía en el voto}

El estudio del comportamiento electoral incluye, al menos, tres tradiciones. La primera se desarrolla en la Universidad de Columbia durante los años cuarenta y asume como planteamiento general que el voto está fuertemente condicionado por la posición del individuo en la estructura social; por lo que las características sociodemográficas, como el género, la edad, el hábitat, la educación, la religión o la clase social, constituirían importantes prescriptores del voto. Posteriormente, la pérdida de importancia de las fracturas sociales como ejes vertebradores de la sociedad (Lipset y Rokkan: 1967), cede protagonismo a la importancia de los procesos de socialización en la adquisición y definición de valores políticos. Para esta escuela, surgida en la Universidad de Michigan, la socialización proveería al individuo de estructuras mentales, como la identificación partidista o la ideología, con las que se decidiría sobre política

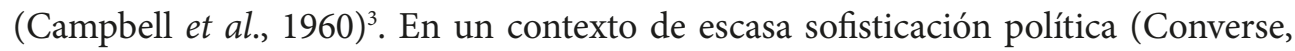
1964; Gramacho, 2006: 4), los electores limitarían además su exposición a noticias y medios que fuesen consistentes con sus predisposiciones políticas (Festinger, 1957), lo que redundaría en un papel limitado de los medios de comunicación ${ }^{4}$ y en un comportamiento electoral poco ligado a la coyuntura. La tercera de las tradiciones, de la

3. Campbell et al. (1960) las denominaron cognitive shortcuts (atajos cognitivos), es decir, esquemas simplificadores de la realidad que facilitan el manejo de mucha información, sin una preparación específica (Popkin, 1991). Estos autores identificaron un atajo cognitivo de gran importancia para la explicación del voto en los Estados Unidos: la identificación partidista. Sin embargo, en España este papel lo cumple la identificación ideológica (Gunther y Montero, 1998).

4. La tradición de los estudios sobre los efectos mínimos o limitados de los medios de comunicación, nace con el célebre estudio de Lazarsfeld et al. (1944) en que se contrastaba empíricamente las visiones convencionales anteriores respecto al potencial de influencia prácticamente ilimitado de los medios de comunicación (parte de estas visiones eran la «teoría de la aguja hipodérmica» o «de la bala mágica»). Estos autores concluyeron que el efecto 
elección racional, se asienta sobre las teorías económicas de la democracia (Downs, 1957; Schumpeter, 1945; Fiorina, 1981; Key, 1966), que advierten de la capacidad del votante para determinar sus preferencias a partir de las potenciales ganancias que le ofrecen las distintas ofertas políticas. Contrariamente a las explicaciones estructurales o psicológicas de las escuelas precedentes, aspectos coyunturales como la evaluación de la situación política o la situación económica, el rendimiento del Gobierno y de la oposición, o el liderazgo (Farrell y Webb, 2002; Shapiro, 1997; Rico, 2002, 2011), serían variables prescriptoras del voto. La pérdida de capacidad explicativa de los factores estructurales también se relaciona con el creciente desencanto social con la política (Norris, 2000; Lorente y Sánchez, 2018), lo que por otro lado responde al creciente proceso de desalineamiento partidista (Dalton, McAllister y Wattenberg, 2002).

Las teorías del voto económico se sitúan en esta tercera escuela, poniendo el acento en elementos evaluativos de la situación económica que se incorporan en la decisión del voto (Downs, 1957). En este sentido, la cobertura mediática sobre las políticas públicas, suele conceder mayor «saliencia» ${ }^{5}$ a la política económica (Balcells-Ventura, 2003: 2). Dos axiomas constituyen la base de las teorías sobre el voto económico. De una parte, según la teoría del mandato (Downs, 1957; Schumpeter, 1945), los partidos políticos presentarán propuestas que generen expectativas sobre la prosperidad económica del país, tratando de conseguir el mayor número de votos posible. De otra, la teoría del cumplimiento o responsabilidad Key $(1966)^{6}$ defiende que los ciudadanos son capaces de evaluar la gestión económica que ha realizado un Gobierno. Para ello, se asume que los grandes indicadores macroeconómicos son fruto de las políticas económicas desarrolladas. Pese a la importancia de estas primeras investigaciones, no sería hasta la década de los años setenta que desde el ámbito académico se comienza a prestar más atención a estos fenómenos (Mueller, 1970; Goodhart y Bhansali, 1970; Kramer, 1971), desarrollándose varios debates propios, como el de si el voto económico depende de la evaluación prospectiva o retrospectiva, es decir, si sobre la decisión de voto influyen las expectativas que generan las propuestas de índole económica de las formaciones políticas, o si lo hace la evaluación de la gestión en materia económica. Mayoritariamente, la corroboración empírica respalda la idea de que el análisis retrospectivo de la situación económica influye más que la evaluación prospectiva (Fiorina, 1981; Nannestad y Paldam, 1995; Lewis-

\footnotetext{
cuantitativamente más importante de la campaña electoral que estudiaron fue el de reforzar las predisposiciones políticas de los votantes, lo que se consideró como efectos limitados.

5. El término "saliencia» procede del inglés saliency y hace referencia a la visibilidad que adquiere un tema respecto a otros.

6. Confrontar Nannestad y Paldman (1994).
} 
Beck, 1988; Key, 1966). En el caso español, es habitual encontrar ambas variables en los modelos aplicados (Bosch, Díaz y Riba, 1999: 182; Fraile, 2002).

Otra discusión versa sobre si los votantes toman en consideración la mejora de su propia situación económica (egotrópico) o la de su país (sociotrópico). En este sentido, el trabajo de Kinder y Kiewit (1979) acaba con la asunción generalizada de la explicación egotrópica del voto, aportando evidencia empírica y base teórica al modelo sociotrópico; dando paso al consenso en torno a la hipótesis del voto sociotrópico. Esta cuestión está muy relacionada con el contexto, puesto que se encuentra muy marcada por aspectos culturales. La aplicación de uno u otro modelo va a depender de los niveles de responsabilidad que se atribuyen al Gobierno respecto de la situación económica personal; cuanto mayor sea ésta, mayor importancia tendrá el voto egotrópico. En España, las investigaciones sobre el tema se decantan por el modelo sociotrópico, utilizándose variables relativas al contexto general (Fraile, 2001, 2002; Maravall y Przeworski, 2001). Si la valoración sobre la situación económica general del país tiene un mayor impacto sobre el voto, ¿cómo se llega a la misma? Parece claro que los medios de comunicación van a jugar aquí un papel clave, por lo que en este trabajo se tiene en cuenta el seguimiento informativo por sus posibles efectos sobre el voto.

La pregunta de si el Gobierno es responsable de la situación económica nuclea otro debate. Aunque el voto económico parte de que se atribuye al Gobierno dicha responsabilidad, dicha imputación puede verse diluida por las circunstancias, de lo que resulta una mayor dificultad para evaluar la gestión económica gubernamental (Hall, 1993). Pueden distinguirse al menos cuatro escenarios en que la atribución de responsabilidades se diluye: los Gobiernos de coalición, la pluralidad de niveles de Gobierno, la existencia de problemas de gobernabilidad (como la dificultad para formar las mayorías necesarias para el desarrollo del proceso legislativo) o la pérdida de soberanía nacional (como la pérdida de instrumentos de política económica que comporta la pertenencia a la Unión Europea). Respecto de estos factores que pueden interferir en la atribución eficaz de la responsabilidad, Fearon (1999) sugiere que la conexión entre accountability ${ }^{7}$ y responsabilidad gubernamental no es automática. Por otra parte, la tesis sobre la calidad de la responsabilidad (Powel y Whitten, 1993) sostiene que cuando los sistemas institucionales permiten identificar más fácilmente a los responsables políticos, la importancia del voto económico es mayor. España presenta un escenario institucional muy confuso para la atribución de responsabi-

7. El concepto accountability se refiere a rendición de cuentas. O’Donnell (1998) añadió a la dimensión de accountability vertical (la que realizan los ciudadanos por medio de elecciones, castigando a o premiando a los líderes), la de accountability horizontal (autocontrol del Estado por medio de agentes estatales con autoridad para evitar conductas ilícitas). 
lidades, por su pertenencia a la Unión Europea y el engranaje institucional multinivel (Lago y Lago, 2011). Además, el voto económico tiene menor impacto en países como España, en que los partidos políticos están consolidados socialmente (Maravall y Przeswoski, 2001; Gramacho, 2006: 4). En el gráfico 1 se muestran los resultados de preguntar a los españoles acerca del actor fundamental en la responsabilidad de la crisis económica. Sólo tres de cada diez entrevistados consideraban que el Gobierno fue el actor con mayor responsabilidad. El sistema bancario obtuvo el segundo puesto, seguido del contexto económico internacional.

\section{Gráfico 1}

Responsabilidad principal sobre la crisis económica en España (\%)

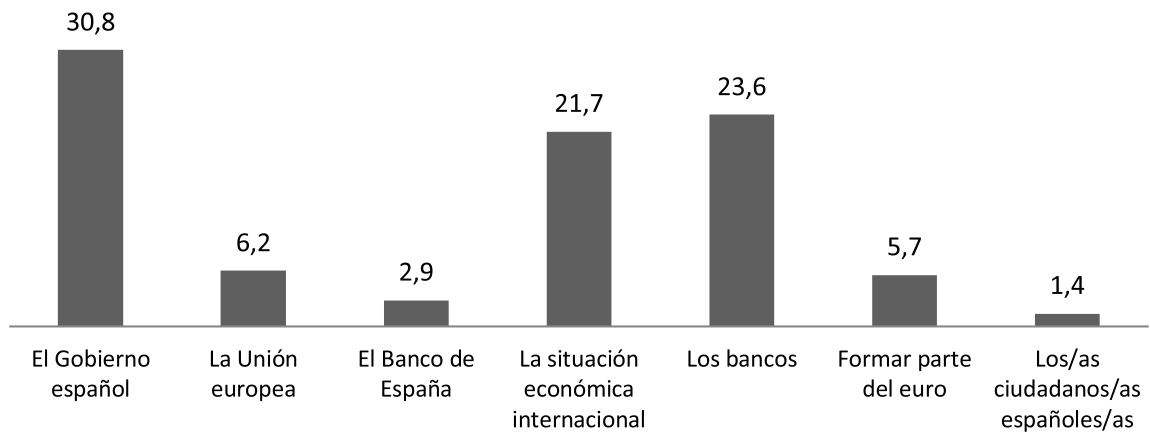

Fuente: Elaboración propia a partir de datos del estudio poselectoral de las elecciones generales de 2011, del CIS, № 2920. Resto hasta sumar 100 corresponde a las categorías "no sabe" y "no contesta".

Además de las dificultades para atribuir la responsabilidad, las expectativas de gestión de la economía respecto a las formaciones políticas en la oposición pueden ser peores que la evaluación de la gestión del Ejecutivo (pese a que ésta no sea positiva). En este sentido, Sanders (1996 y 2000), que incluye la influencia de las alternativas al Gobierno y de sus propuestas en la hipótesis de la responsabilidad, afirma que si la opinión pública valora negativamente la alternativa política al Gobierno, la probabilidad de que los electores cambien su voto, disminuye. Este planteamiento, que se incluirá en los estudios sobre voto económico posteriores ${ }^{8}$, resulta útil para nuestro análisis. También relacionada con esta cuestión se encuentran las llamadas «funciones de popularidad» o policy-oriented voting (Kiewiet, 1981), que apoyan la tesis de que el voto se orienta, en parte, por la reputación de cada partido para 
resolver cada tipo de problema. La idea de que hay una serie de temas o políticas públicas en las que destacan los diferentes partidos políticos, tiene su origen en el nacimiento de las organizaciones partidistas, en tanto que articuladoras de intereses. También en España, unas formaciones políticas abordan con mayor comodidad los temas de políticas sociales, mientras que a otras les ocurre lo propio al tratar asuntos de gestión económica o seguridad. Por tanto, si no se identifica a las formaciones políticas en la oposición con una gestión de la economía eficaz, es muy posible que no se produzca el castigo al partido en el Gobierno. Downs (1973: 43) ya se refería a la «diferencia esperada de partido» para señalar que era importante en la decisión del voto la diferencia entre la utilidad percibida durante el período de Gobierno y la que se estima que habría habido en el caso de haber estado la oposición en el Gobierno.

Al mencionar el policy-oriented voting, se ha puesto de manifiesto que tradicionalmente, los estudios sobre voto económico han ignorado las valoraciones respecto a la gestión de otras políticas públicas. Sin embargo, los programas económicos de los Gobiernos, especialmente en el caso de los socialdemócratas, incluyen también políticas sociales (Fraile, 2005), que además pueden constituir un instrumento al que se recurre en circunstancias de crisis económica. De ahí que en diferentes investigaciones se haya incorporado la valoración de la gestión en distintas políticas públicas. Para el caso español, esta hipótesis ya ha sido corroborada (Maravall y Fraile, 2000; Fraile, 2005).

La propuesta de las funciones de popularidad también abrió el debate en torno a si la influencia la ejercía la situación económica objetiva (datos de los macro indicadores económicos), o la percepción social de la situación económica. Los estudios iniciales sobre voto económico, utilizaban variables sencillas de datos económicos agregados, como la tasa de paro o la inflación. También se utilizaron datos relativos al producto interior bruto, el poder adquisitivo o la presión fiscal. Fiorina (1981) señaló la necesidad de medir las preocupaciones e impresiones de la ciudadanía sobre la economía. Sin embargo, Sanders, Ward, Marsh y Fletcher (1987), apostarían definitivamente por las variables objetivas, dado que éstas mostraban una relación mucho mayor con el voto. En este sentido, si las fuentes de información pueden ser, a grandes rasgos, la agenda mediática y el contexto de la experiencia cotidiana individual; y el elector incorpora más el juicio sociotrópico que el egotrópico, los medios desempeñarían un papel clave. Ahora bien, si se apuesta por el efecto de la agenda mediática, también es preciso asumir que los medios no hacen sino proyectar visiones de la realidad, informar sobre aspectos de la realidad. En definitiva, los datos económicos objetivos influirían sobre la formación de la agenda mediática, que a su vez influiría en las percepciones de los electores. 
Por tanto, la agenda mediática puede ser un elemento del contexto muy relacionado con el voto económico, como también ocurre con la estructura institucional de los sistemas políticos (Duverger, 1951).

Los estudios sobre voto económico para el caso español han ido en aumento en las últimas décadas, aunque no es hasta la de los noventa que comienza a adquirir especial relevancia. Por su aporte teórico, destacaríamos los estudios de Maravall y Przewoski (2001)9 , y la revisión realizada por Gramacho (2006). También destaca el compendio teórico elaborado por Bosch, Díaz y Riba $(1999)^{10}$. Clave para este trabajo ha sido la investigación de Fraile (2005), que puede ser el análisis más profundo y riguroso sobre voto económico en España.

Dos estudios han sido determinantes para el planteamiento metodológico de este trabajo. Por un lado, el estudio de Bosch y Riba (2005), en que se elabora un modelo de popularidad para el PSOE, en los años en que estuvo en el Gobierno. Entre sus principales resultados se encuentra el hallazgo de significación estadística en las relaciones con el voto de las variables desempleo, inflación, expectativas económicas y liderazgo. Además, el cálculo de mediciones se lleva a cabo utilizando retardos estadísticos, lo que mejora el ajuste y control del efecto temporal entre variables. Por otro lado, el estudio de Fraile (2002) que tiene por objeto analizar la importancia de la percepción de la gestión económica sobre el voto, donde propone un modelo que denomina «de voto extendido», en que se tiene en cuenta la importancia de los contextos políticos y sociales. Es un modelo novedoso por dos razones: porque se incluye la valoración de la política económica, en lugar de la situación económica; y porque incorpora la evaluación de la política social ${ }^{11}$. Por último, este estudio aplica estrategias metodológicas para controlar la racionalización en los juicios sobre la economía (Maravall y Przewoski, 2001). En general, en todos los estudios sobre comportamiento electoral en España, se incluye el análisis de los efectos de la economía en el voto. Para el estudio que aquí se plantea, sobre dos elecciones generales en España, es preciso describir brevemente el contexto de los comicios objeto de análisis.

9. Maravall y Przeworski (2001) llegan a la conclusión de que la relación entre el voto y los juicios prospectivos y retrospectivos sobre la economía, no responde a una lógica de comportamiento mecánica, como sostiene la teoría clásica. Sugieren que, en muchas ocasiones, los juicios sobre la economía responden a consideraciones políticas previas.

10. En este artículo se ha seguido este trabajo para la presentación de los principales debates teóricos relacionados con el voto económico.

11. Fraile (2002) constata que las políticas sociales tienen consecuencias electorales. Cfr. Fraile (2001), González (1996), Maravall y Fraile (2000). 


\section{La situación económica en España: de la bonanza de 2000 a la crisis de 2011}

Para contextualizar brevemente las elecciones analizadas, haremos referencia a indicadores económicos y a datos de carácter actitudinal, procedentes en el primer caso del Instituto Nacional de Estadística y en el segundo del CIS.

Debemos hacer referencia en primer lugar a uno de los problemas estructurales de España, el desempleo. Desde la crisis económica mundial de la década de los setenta y que no se supera hasta iniciarse la de los ochenta, el paro en España nunca se ha situado por debajo de la barrera del $8 \%$, lo que tradicionalmente lo ha colocado como el país de la OCDE con mayor nivel de desempleo. De hecho, la tasa española de paro ha doblado la media de los países desarrollados de su entorno; tanto en épocas de expansión económica, como en fases de contracción de ciclo. A pesar de ser muchos los factores que pesan en el desarrollo del desempleo en España, es clave el carácter intensivo en mano de obra del modelo productivo, con un tejido empresarial que aportaba escaso valor añadido, si lo comparamos con niveles europeos. Esta dependencia de la mano de obra se agrava con la denominada «burbuja inmobiliaria», que acelera el ritmo de la construcción en España desde mediados de la década de los noventa, convirtiendo así al sistema de trabajo nacional en una pieza peligrosamente vulnerable de la coyuntura económica, cuyo desenlace es ya conocido, a partir de la crisis financiera internacional que se desencadena a mediados de 2007. De 1994 a 2007, España experimenta una fuerte y prolongada expansión económica. Uno de los factores principales que la favorece es la entrada del país en la Unión Monetaria, que provocó el incremento de la confianza financiera en España, así como un importante descenso de los tipos de interés. En gran medida, la conjunción de ambos factores produjo la continua demanda de créditos para la compra de inmuebles por particulares y empresas, que incrementó el trabajo para mano de obra poco cualificada en el sector de la construcción.

Como cabría esperar, la cuestión del desempleo ha sido objeto incesante de preocupación para los españoles. El gráfico 2 muestra la evolución del lugar que se otorga a «el paro» $\mathrm{y}$ «los problemas de índole económica» en la pregunta relativa a los tres principales problemas de España. Los valores son aproximadamente constantes desde 2000 a 2007, incluso con un ligero descenso de «el paro» como principal problema, situándose por debajo del 40\%, a principios de 2007. Del mismo modo, se aprecia el más que notable aumento de la percepción de ambas cuestiones como problemáticas a partir del estallido de la crisis económica mundial, a mediados del año 2007, cuyos valores para el caso de «el paro» se sitúan por encima del $80 \%$ a las puertas de las elecciones generales de noviembre de 2011, en que el PP, liderado por Mariano Rajoy, desbanca a los socialistas de Zapatero tras dos legislaturas en el Gobierno (2004-2011). 
Gráfico 2

Principales problemas que existen en España (\%)

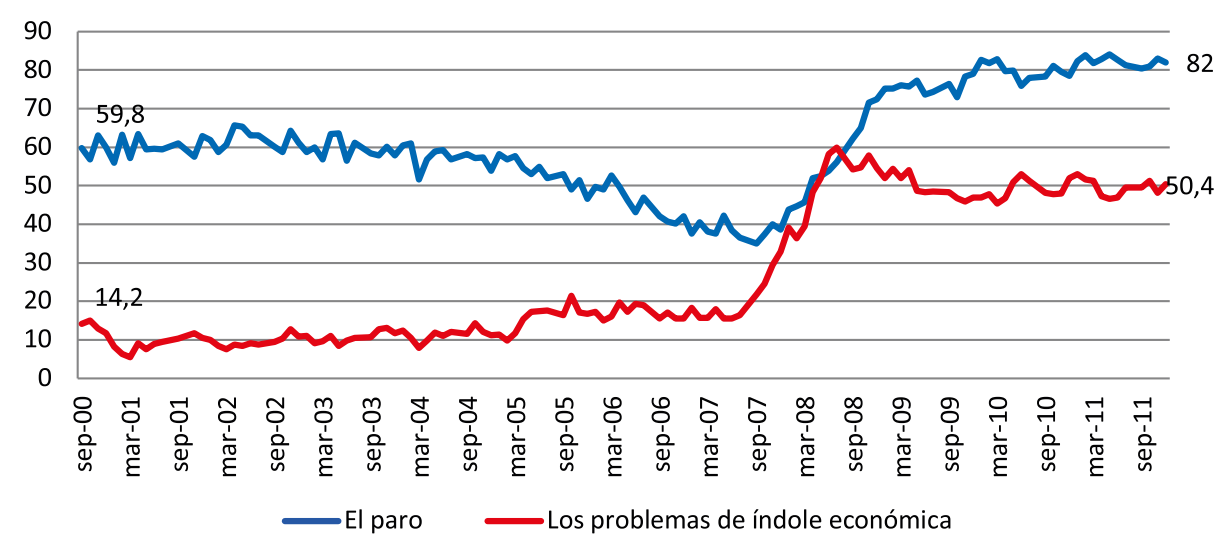

Fuente: Elaboración propia a partir de datos del Centro de Investigaciones Sociológicas y el INE.

Las percepciones juegan un papel preferente en las teorías sobre voto económico. Las percepciones negativas de la economía activan los procesos de racionalización y de activación de conductas que podríamos denominar «utilitaristas». El gráfico 3 muestra la evolución de las expectativas relacionadas con la situación económica ${ }^{12}$. Puede apreciarse cómo las expectativas acerca de la situación económica se mantienen en una curva bastante inestable, con cierta estacionalidad aparente, que comienza a descender en 2005, se desploma durante el año 2008 y se recupera desde mediados de 2009, aunque sin llegar a los niveles del lustro anterior.

Llegados a este punto, se impone la pregunta de cómo se relaciona la realidad económica con las percepciones de la misma. Más interesante para nuestro estudio, si existe relación entre la realidad económica y las valoraciones que se hacen del Gobierno. Para tratar de responder al primer interrogante, el gráfico 4 muestra la evolución de la tasa de paro y la percepción negativa («mala»o «muy mala») de la situación económica ${ }^{13}$. Se observa que la relación entre ambas variables es notable,

12. El Indicador de Expectativas Económicas (IEE) se construye a partir de la pregunta «¿Cree que dentro de un año la situación económica del país será mejor, igual o peor?», con la siguiente ecuación:

$M E=\frac{100 p_{1}+50 p_{3}+0 p_{2}}{p_{1}+p_{2}+p_{3}}$ donde $\mathrm{p}_{1}, \mathrm{p}_{2}$ y $\mathrm{p}_{3}$ son, las proporciones de respuesta de las opciones mejor, igual y peor, respectivamente.

13. Se observa que la valoración de la situación económica de España se mantiene en valores estables hasta mediados de 2007, momento en que la valoración negativa de la situación económica inicia un progresivo y continuo crecimiento, hasta situarse por encima del 80\% desde inicios de 2011. 
con un coeficiente de correlación de Pearson de 0.84 . Nótese que la relación es prácticamente perfecta desde el inicio de la crisis, en el año 2007, lo que puede indicar que la relación entre lo objetivo y lo perceptivo es más importante en situaciones de crisis económica. Parece que, tal y como se indicaba en la teoría, los electores castigan más que premian por la situación económica.

\section{Gráfico 3}

Valoración de la situación económica prospectiva

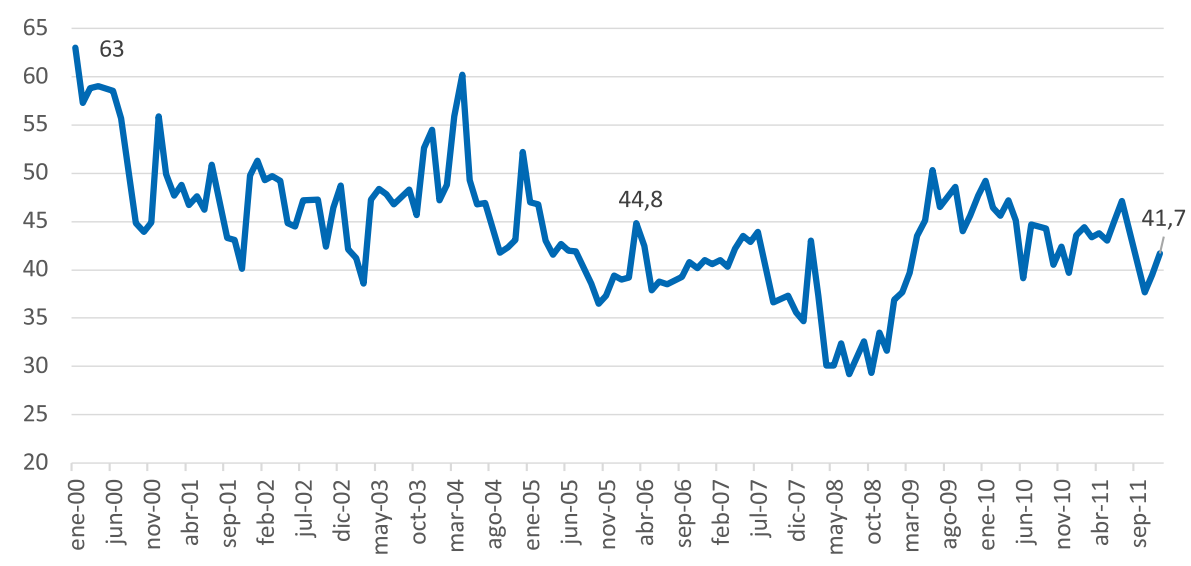

Fuente: Centro de Investigaciones Sociológicas.

\section{Gráfico 4}

Evolución de la tasa de desempleo y de la valoración negativa de la situación económica actual

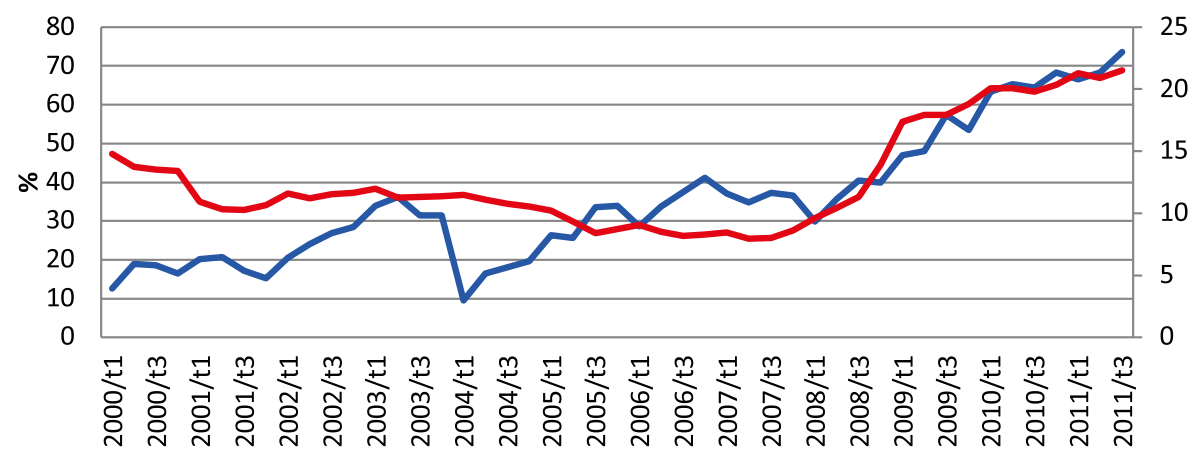

- Valoración situación actual económica como mala o muy mala —Tasa de paro 
Respecto al segundo de los interrogantes, sobre la relación entre los datos objetivos y la popularidad del Gobierno (medida a través de una pregunta de valoración), en el gráfico 5 se observa una relación muy semejante a la anterior. La correlación entre variables, para el período 2000-2011, es de 0.81. Ahora bien, si se divide esta etapa en dos, de 2000 a 2004 y de 2004 a 2011, se aprecian grandes diferencias. Durante el primer período (2000-2004), valorado como positivo en lo económico, la relación entre economía objetiva y popularidad del Gobierno del PP es de 0.27, lo cual es una correlación muy baja. En cambio, de 2004 a 2011, la correlación es de 0.93, prácticamente una correlación positiva perfecta: a mayor tasa de paro, peor valoración del Gobierno del PSOE. Se ponen de manifiesto las diferencias en los efectos de la economía en la popularidad del Gobierno.

\section{Gráfico 5}

Evolución de la tasa de desempleo y de la valoración negativa del Gobierno

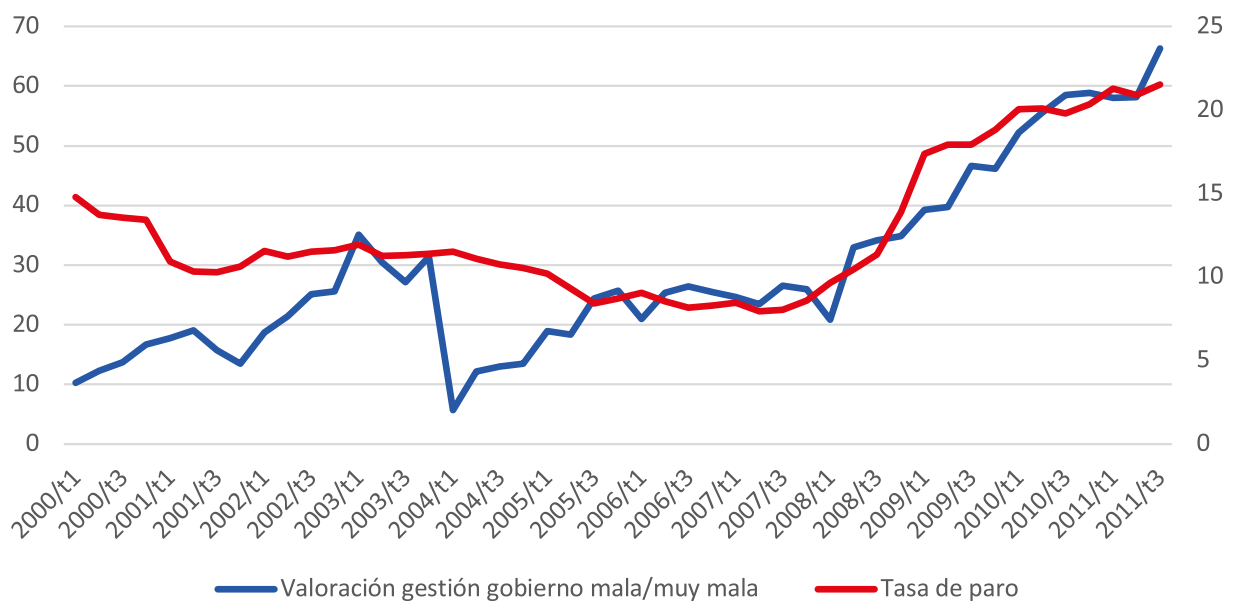

Fuente: Centro de Investigaciones Sociológicas.

El caso español no difiere de las conclusiones de los estudios sobre voto económico. En las próximas páginas se expondrán algunos aspectos clave para el planteamiento metodológico del análisis de las relaciones entre economía y voto, especialmente en lo que a definición y operacionalización de variables se refiere. 


\section{La influencia de la economía en el comportamiento electoral de los españoles: aspectos metodológicos}

Esta sección se dedica a someter a prueba la hipótesis de la asimetría del voto económico, para las elecciones generales de los años 2000 y 2011.

Para el análisis, utilizaremos la siguiente ecuación de explicación del voto, que sintetiza el modelo de voto económico defendido en este estudio:

$Y=($ Expectativas económicas + Valoración de la política económica del Gobierno

+ Valoración de la hipotética gestión de la economía por la oposición + Política social + Consumo informativo + Variables de control $+\varepsilon$ ).

La variable dependiente (y) es la intención de votar al partido en el Gobierno. Dado que la técnica de análisis que se aplicará será la regresión logística binomial, la variable dependiente se ha codificado tomando el valor 1 para la intención de voto al partido en el Gobierno (PP en 2000 y PSOE en 2011) y 0 para el resto de categorías (incluidos los que responden que no votarán, «no sabe» 0 «no contesta»).

Respecto a las variables de tipo económico, se incluyen las «expectativas económicas», que toman el valor 0 cuando el encuestado considera que la situación económica irá a peor o mucho peor, 1 si el entrevistado opina que será igual y 3 cuando estima que la situación económica será mejor o mucho mejor. La variable «valoración de la política económica del Gobierno» toma el valor 0 cuando la valoración es mala o muy mala, 1 si es regular y 2 cuando es buena o muy buena. En cuanto a la variable "evaluación de la hipotética gestión de la economía por la oposición», toma el valor 0 cuando el encuestado considera que el Gobierno lo haría mejor y 1 si considera que la oposición la gestionaría mejor.

La variable «partido que mejor gestiona la política social» se ha codificado como un índice de cuatro niveles, que combina las políticas de empleo, sanidad y educación, y que toma el valor 0 cuando el encuestado considera que el partido en el Gobierno gestiona mejor las tres políticas, el valor 1 si el entrevistado entiende que el Gobierno gestiona mejor que la oposición, al menos dos políticas públicas, el valor 2 cuando la percepción es que la oposición gestionaría mejor que el Gobierno, al menos dos políticas públicas y el valor 3 si se opina que la oposición gestionaría mejor que el Gobierno las tres políticas. Asimismo, en consonancia con el concepto de la racionalidad limitada (Simon, 1957) ${ }^{14}$, se ha introducido un índice relativo a

14. El concepto de racionalidad limitada suele explicarse como una aportación para superar las limitaciones del modelo racionalista para el análisis de las políticas públicas. El racionalismo aboga por la búsqueda de la solución óptima, teniendo en cuenta toda la información, lo que lo convierte en un modelo de toma de decisiones, cuando menos, poco realista. La propuesta de Simon (1947) aboga por la búsqueda de una solución ya no óptima, sino satisfactoria, en la búsqueda de una racionalidad limitada por las condiciones que impone la realidad. 
consumo informativo, que combina la frecuencia del seguimiento de la información política a través de televisión, radio y prensa, y que oscila entre el valor 0 para «bajo seguimiento» y 6 por «alto seguimiento».

Por último, se incluyen las variables de control convencionales en los estudios sobre los determinantes del voto a nivel individual: como la «auto-ubicación ideológica» en la escala de uno a diez, que permitirá controlar que los juicios sobre la economía no sean racionalizaciones ex post de las predisposiciones políticas; el «sexo», codificada como 0 para mujeres y 1 para hombres; la «edad», que se incluye como variable cuantitativa; la «educación», como variable métrica de cinco niveles y la "ocupación», que contrapone a los encuestados que trabajan con los jubilados y pensionistas, parados, estudiantes y los dedicados a las labores del hogar.

Los datos utilizados en este estudio proceden de las encuestas con estructura de panel realizadas por el CIS. En la tabla 1 se resumen los datos técnicos principales.

Tabla 1

Estudios demoscópicos explotados para el análisis

\begin{tabular}{ccccc}
\hline Estudio & No Estudio & $\begin{array}{c}\text { Tamaño } \\
\text { muestral }\end{array}$ & $\begin{array}{c}\text { Error } \\
\text { muestral }\end{array}$ & Fecha de realización \\
\hline $\begin{array}{c}\text { Preelectoral Elecciones } \\
\text { Generales 2000 }\end{array}$ & 2382 & 24040 & $\pm 0,64 \%$ & $\begin{array}{c}\text { Del 11 al 28 de febrero } \\
\text { de 2000 }\end{array}$ \\
\hline \hline $\begin{array}{c}\text { Postelectoral Elecciones } \\
\text { Generales 2000 }\end{array}$ & 2384 & 5283 & $\pm 1,34 \%$ & $\begin{array}{c}\text { Del 18 de marzo al } 18 \text { de } \\
\text { abril de 2000 }\end{array}$ \\
\hline $\begin{array}{c}\text { Preelectoral Elecciones } \\
\text { Generales 2011 }\end{array}$ & 2915 & 17194 & $\pm 0,76 \%$ & $\begin{array}{c}\text { Del } 6 \text { al 23 de octubre } \\
\text { de 2011 }\end{array}$ \\
\hline $\begin{array}{c}\text { Postelectoral Elecciones } \\
\text { Generales 2011 }\end{array}$ & 2920 & 6082 & $\pm 1,3 \%$ & $\begin{array}{c}\text { el 24 de noviembre } \\
\text { de 2011 al 15 de enero } \\
\text { de 2012 }\end{array}$ \\
\hline \hline
\end{tabular}

Fuente: Elaboración propia a partir de las fichas técnicas diseñadas por el CIS.

La técnica estadística que se emplea para el análisis, es la regresión logística binaria, donde los parámetros se estiman a través del método de máxima verosimilitud. Además de analizar los resultados del análisis de regresión, se calculan funciones de probabilidad siguiendo la fórmula:

$$
\mathrm{P}_{\mathrm{i}}=\frac{\mathrm{e}^{\mathrm{y}}}{1+\mathrm{e}^{\mathrm{y}}}=\mathrm{P}_{\mathrm{i}}=\frac{1}{1+\mathrm{e}^{-\mathrm{y}}}
$$

Siendo «y» la función lineal del modelo de regresión logística, y «e» la base de logaritmos neperianos. 


\section{Resultados: el modelo de voto económico en las elecciones de 2000 y 2011}

Los resultados del análisis del voto económico en las elecciones de $2000^{15}$ y $2011^{16}$, calculados con la técnica del logit binomial, se presentan en la tabla 2. En la primera columna aparecen las variables independientes. En la segunda y cuarta columnas, se muestran los coeficientes beta de regresión, acompañados entre paréntesis de los errores típicos correspondientes, así como el nivel de significación estadística. Finalmente, en la tercera y quinta columnas aparecen los valores odds ratio asociados a cada variable ${ }^{17}$.

Los resultados del modelo de voto de las elecciones de 2000 (tabla 2) dan cuenta de la relevancia del voto económico en dichos comicios. Las tres variables económicas son estadísticamente significativas, en el sentido esperado. Así, la probabilidad de votar al PP frente al resto de opciones, se incrementa cuando el entrevistado presenta expectativas optimistas sobre la situación económica y valora positivamente la política económica. La variable sobre la hipotética gestión de la política económica por la oposición, presenta un coeficiente negativo, como cabía esperar. En el caso de la valoración del partido que mejor gestionaría la política social y el nivel de seguimiento de la información política, el análisis parece reflejar la falta de significatividad estadística de dichas variables. La ideología presenta significatividad estadística en el sentido esperado, mostrándose una mayor probabilidad de voto al partido en el Gobierno, cuanto más a la derecha se ubique el entrevistado. Concretamente, el aumento de una unidad en la ubicación ideológica si el resto de variables se mantuvieran constantes, aumentaría los odds de votar al partido popular 1,805 veces más que si no se aumentara esa unidad en la escala ideológica. Ninguna de las variables sociodemográficas incluidas como variables de control en el análisis, resulta estadísticamente significativa.

Los resultados del modelo de voto de las elecciones de 2011, evidencian un protagonismo menor de las variables económicas en la probabilidad de voto al ejecutivo socialista. En cuanto a la variable de expectativas económicas (tabla 2), presenta signo negativo, lo que indica que cuanto más positivas sean las expectativas, menor será la probabilidad de voto al PSOE. Bajo la lógica del voto económico, esta cuestión resulta inconsistente, pues sería razonable según la teoría de la elección racional, que un elector con altas expectativas económicas decidiese votar al partido en el Gobierno. Sin embargo, es preciso matizar, en primer lugar, que a pesar de que la pregunta sobre expectativas económicas tradicionalmente se mide en el estudio preelectoral, en este caso se sitúa en el estudio poselectoral. A ello se le suma otra cuestión, y es que las elecciones de 2011 se presentaban con baja incertidumbre, pues los sondeos

15. El modelo aplicado para el año 2000 predice correctamente cerca del $86 \%$ del total de observaciones (932 casos). El coeficiente de determinación Pseudo $\mathrm{R}^{2}$ alcanza el 0,7.

16. El modelo del año 2011 predice correctamente el 88\% de los casos observados (2251). El coeficiente de determinación Pseudo $\mathrm{R}^{2}$ roza el 0,7 .

17. Se realizaron análisis de multicolinealidad entre variables, sin detectarse problemas. 
preelectorales dibujaban un escenario beneficioso para la alternativa al partido en el poder, por lo que unas expectativas económicas positivas podrían estar asociadas al ánimo generalizado de que se produciría un cambio en el partido de Gobierno. En este sentido, si se cruzan las expectativas económicas y la intención de voto, puede observarse que una mayoría de los entrevistados que responden con expectativas positivas, son potenciales votantes del PP.

Respecto a la valoración de la política económica del Gobierno, el análisis muestra una mayor probabilidad de voto al PSOE cuanto mejor se valore la política económica. Observamos, sin embargo, que el peso marginal de esta variable es comparativamente inferior al que ofrece en el modelo de 2000, lo que podría explicarse porque sólo un $2.4 \%$ de los encuestados valoran como buena o muy buena la gestión económica del Gobierno. En cuanto a la percepción sobre quién gestionaría mejor la economía, la variable es estadísticamente significativa al 90\%, en el sentido esperado. Sin embargo, en el estudio preelectoral sólo un $15,1 \%$ de los entrevistados estima que el PSOE es el partido que mejor gestiona la economía. En lo que se refiere a la valoración del partido que mejor gestionaría la política social, el modelo presenta un coeficiente estadísticamente significativo, contrariamente a lo que ocurría en el modelo de 2000, y en la dirección esperada: la probabilidad de votar al PSOE entre quienes consideran que el Gobierno es el que mejor gestiona la política social ${ }^{18}$, se incrementa notablemente, lo que la convierte en una variable clave en la explicación del voto al PSOE en el año 2011. Mientras que sólo algo más de la mitad de los votantes socialistas creía que el PSOE era el que mejor podía gestionar la economía, dicha cifra asciende al $87 \%$ cuando se trata de la gestión de la política social.

En cuanto a las variables de control, la ideología se comporta como cabía esperar, reduciéndose la probabilidad de votar al PSOE por cada aumento de una unidad en la ubicación en la escala ideológica de los entrevistados. El nivel educativo también presenta signo negativo, es decir, a mayor nivel de formación, menor probabilidad de votar al PSOE. En cuanto a la edad, presenta una significación del $90 \%$, con signo negativo.

La tabla 3 permite comparar, mediante simulaciones calculadas a partir de los coeficientes de los modelos expuestos en la tabla 2, la diferencia de magnitud de la importancia del voto económico en las dos elecciones. En la primera columna aparecen las variables de interés y sus categorías mínimas y máximas. En la segunda y tercera columnas, la probabilidad de voto al partido en el Gobierno para cada elección. Se ha calculado las probabilidades para las categorías extremas de cada una de las variables, manteniendo estables el resto de variables en los valores promedios de su distribución muestral ${ }^{19}$.

18. Recuérdese que la variable «partido que mejor gestiona la política social» combina la política de empleo, la política sanitaria y la de educación.

19. En el cálculo de probabilidades del año 2000, los valores promedio de las variables caracterizan un elector con expectativas económicas moderadas: una valoración moderada de la gestión económica del gobierno; la per- 
La influencia de la economía en el voto en España: de la bonanza de 2000 a la crisis de 2011

Tabla 2

El voto económico en las elecciones del 2000 y 2011

\begin{tabular}{|c|c|c|c|c|}
\hline \multirow[b]{2}{*}{ Variables independientes } & \multicolumn{2}{|c|}{ Elecciones Generales de 2000} & \multicolumn{2}{|c|}{ Elecciones Generales de 2011} \\
\hline & Beta & $\operatorname{Exp}(B)$ & Beta & $\operatorname{Exp}(B)$ \\
\hline Expectativas económicas & $0,588(0,172) * *$ & 1,801 & $-0,304(0,112) *$ & ,738 \\
\hline $\begin{array}{l}\text { Valoración de la política económica } \\
\text { del Gobierno }\end{array}$ & $0,844(0,199) * *$ & 2,326 & $0,626(0,129) * *$ & 1,871 \\
\hline $\begin{array}{l}\text { Valoración de la hipotética gestión } \\
\text { de la economía por la oposición }\end{array}$ & $-3,625(1,348) *$ & 0,027 & $-0,413(0,230)+$ & ,662 \\
\hline Política social & $-0,443(0,305) \mathrm{ns}$ & 0,642 & $-1,309(0,127) * *$ & ,270 \\
\hline Consumo informativo & $0,079(0,052) \mathrm{ns}$ & 1,083 & $0,062(0,041) \mathrm{ns}$ & 1,064 \\
\hline \multicolumn{5}{|l|}{ Variables de control } \\
\hline Ideología & $0,590(0,070) * *$ & 1,805 & $-0,240(0,053) * *$ & ,786 \\
\hline Nivel educativo & $0,095(0,091) \mathrm{ns}$ & 1,100 & $-0,425(0,080) * *$ & ,654 \\
\hline Edad & $-0,003(0,009) n s$ & 0,997 & $-0,012(0,007)+$ & ,988 \\
\hline Género & $0,135(0,241) \mathrm{ns}$ & 1,145 & $0,002(0,160) n s$ & 1,002 \\
\hline Jubilado_pensionistas & $0,342(0,349) \mathrm{ns}$ & 1,408 & $0,200(0,264) n s$ & 1,221 \\
\hline Parado & $0,478(0,388) \mathrm{ns}$ & 1,613 & $0,300(0,201) n s$ & 1,350 \\
\hline Estudiante & $0,055(0,475) \mathrm{ns}$ & 1,056 & $-0,103(0,363) \mathrm{ns}$ & ,902 \\
\hline Trabajo_doméstico & $0,317(0,360) \mathrm{ns}$ & 1,372 & $0,643(0,339)+$ & 1,902 \\
\hline Constante & $-5,292(0,757) * *$ & 0,005 & $2,917(0,491) * *$ & 18,484 \\
\hline Chi cuadrado & $682,741^{* *}$ & & 1343,62 & \\
\hline Pseudo $R^{2}$ & ,693 & & 0,666 & \\
\hline \% Correcto & 85,7 & & 88,1 & \\
\hline № casos & 932 & & 2251 & \\
\hline
\end{tabular}

Variable dependiente es 1 (voto por el PP en 2000 y PSOE en 2011) y 0 (no voto por el PP en 2000 y no voto por el PSOE en 2011).

Nota: los datos son coeficientes logit binominal, estimados a través de la función de máxima verosimilitud. Entre paréntesis se incluyen los errores asociados.

** Significativo al nivel del $99 \%$.

* Significativo al nivel del $95 \%$.

+ Significativo al nivel del $90 \%$.

ns No significativo. 
De acuerdo con la tabla 3, la diferencia de probabilidad de voto al Gobierno en función de si el votante presentaba juicios optimistas o pesimistas, fue de 26.68 puntos en el caso del las elecciones de 2000 y de apenas 3.72 puntos en el caso de 2011. Igualmente, la probabilidad de voto al Gobierno entre quienes valoran positivamente la gestión económica del Gobierno y quienes la valoran negativamente, presenta un diferencial de 37.4 puntos en el caso de las elecciones de 2000 y de poco más de 13 puntos en las elecciones de 2011. Escenario parecido presenta la evaluación sobre qué partido gestiona mejor la política económica; de nuevo se observa una mayor relevancia en las elecciones de 2000 que en las de 2011, donde las diferencias entre quienes consideran que el Gobierno la gestiona mejor y quienes consideran que es la oposición quien la gestiona mejor, es de apenas 3 puntos. En cuanto a la gestión de la política social, esta variable (tabla 2 ) no presentaba significatividad estadística en la explicación del voto al PP en el año 2000, mientras que se observa que jugó un papel clave en el voto a los socialistas, presentando un diferencial de probabilidad de cerca de 47 puntos entre quienes consideran que el PP gestiona mejor la política social ${ }^{20}$ y quienes confían más en los socialistas para su gestión. Por último, la ideología presenta una alta capacidad explicativa en ambos comicios y para ambos partidos, con un diferencial de probabilidad muy llamativo para el voto al PP en el año 2000 (86.5 puntos).

En el gráfico 6 se compara el impacto de las expectativas económicas y de la valoración de la hipotética gestión de la economía por la oposición, para cada una de las elecciones. Como puede apreciarse, en ambos comicios el efecto de las expectativas económicas resulta superior a la evaluación de la hipotética gestión de la economía por la oposición, siendo su impacto marginal muy superior en las elecciones del año 2000, con una tasa de variación entre quienes son optimistas y quienes piensan que la situación será igual, de un $+39.31 \%$. En el caso de la valoración de la hipotética gestión de la economía por la oposición, la tasa de variación entre quienes piensan que la oposición lo haría mejor, respecto a la probabilidad estimada para quienes piensan que el gobierno lo haría mejor, es del -95.86\% en el caso de 2000 y del $-31.65 \%$ en el caso de 2011.

cepción de que el gobierno gestiona mejor la política económica; la creencia de que el gobierno gestionaría mejor que la oposición, al menos, dos políticas públicas sociales; 2 en la escala de consumo informativo; ubicado en el cinco ideológico; hombre; de 46 años de edad; jubilado o pensionista y con 3 en la escala de estudios. En el cálculo de probabilidades del año 2011, la referencia difiere en que el elector de referencia tiene 48 años de edad; 2 en la escala de estudios; 2 en el índice de medios; la percepción de que la oposición gestionaría mejor que el gobierno, al menos, dos políticas públicas; considera que la oposición gestionaría mejor la política económica y valora negativamente la gestión económica del gobierno.

20. En las elecciones de 2011, el peso de la política social es similar en el voto al PSOE y al PP, lo que podría estar relacionado con la pérdida de reconocimiento que adquieren los socialistas en dicha materia, tras las políticas de contención del gasto (recortes y reformas restrictivas en materia social), llevadas a cabo por el ejecutivo de Zapatero a final de legislatura. 


\section{Tabla 3}

Efectos de las expectativas económicas, la valoración de la política económica del gobierno, la valoración de la hipotética gestión económica de la oposición, la gestión de la política social

y la ideología en la probabilidad de votar al partido en el gobierno frente a no votarlo, en las elecciones generales de 2000 y 2011

\begin{tabular}{|c|c|c|}
\hline & $\begin{array}{c}\text { Elecciones de } 2000 \\
\text { Probabilidad de voto al } \\
\text { PP }\end{array}$ & $\begin{array}{c}\text { Elecciones de } 2011 \\
\text { Probabilidad de voto al } \\
\text { PSOE }\end{array}$ \\
\hline \multicolumn{3}{|l|}{ Expectativas económicas } \\
\hline Pesimista... & 24,23 & 8,58 \\
\hline Optimistas... & 50,91 & 6,48 \\
\hline Diferencia & 26,68 & 2,1 \\
\hline \multicolumn{3}{|c|}{ Valoración de la política económica del Gobierno } \\
\hline Positiva... & 57,25 & 19,51 \\
\hline Negativa... & 19,85 & 6,48 \\
\hline Diferencia & 37,4 & 13,03 \\
\hline \multicolumn{3}{|c|}{$\begin{array}{l}\text { Valoración de la hipotética gestión de la } \\
\text { economía por la oposición }\end{array}$} \\
\hline La oposición gestiona mejor... & 1,51 & 6,48 \\
\hline El Gobierno gestiona mejor... & 36,54 & 11,47 \\
\hline Diferencia & 35,03 & 4,99 \\
\hline \multicolumn{3}{|l|}{ Gestión de la Política social } \\
\hline La oposición gestiona mejor... & & 1,84 \\
\hline El Gobierno gestiona mejor... & Efecto no significativo & 48,71 \\
\hline Diferencia & & 46,87 \\
\hline \multicolumn{3}{|l|}{ Ideología } \\
\hline 1 (Izquierda)... & 5,15 & 15,34 \\
\hline 10 (Derecha)... & 91,68 & 2,04 \\
\hline Diferencia & 86,53 & 13,30 \\
\hline Probabilidad de base & 36,54 & 6,48 \\
\hline
\end{tabular}

Las probabilidades se han calculado manteniendo el resto de variables incluidas en el modelo constantes en el valor medio. 


\section{Gráfico 6}

Efectos de variables económicas sobre la probabilidad de voto al gobierno

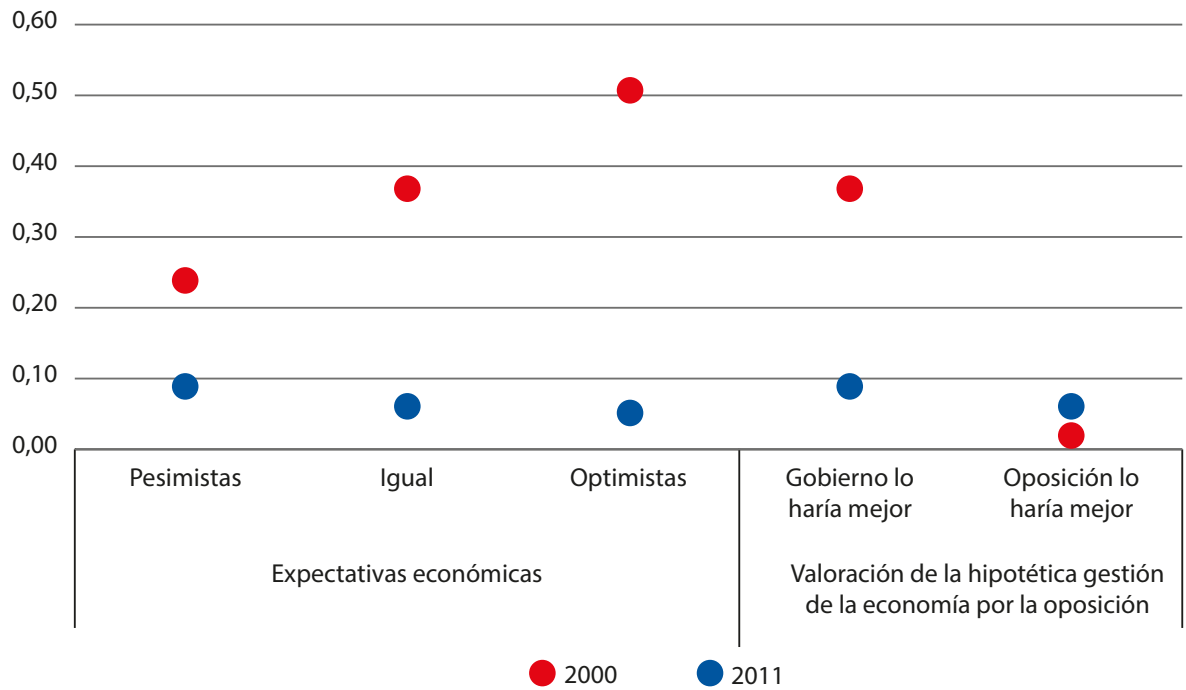

Nota: La puntuación indica el efecto de cada categoría de las variables seleccionadas sobre la probabilidad (tanto por 1) de votar al partido en el gobierno (frente a cualquier otra opción), asumiendo que el resto de variables toman el valor medio de su correspondiente distribución. Todos los valores son estadísticamente significativos.

\section{Conclusiones}

Este trabajo perseguía poner a prueba la hipótesis principal de la asimetría del voto económico, con objeto de aportar nueva evidencia empírica de la relevancia de esta cuestión para los procesos electorales analizados. Como se indica en el apartado metodológico, esta teoría fue refutada para el caso español por Fraile (2002), con resultados que evidenciaban un mayor peso de los factores económicos en el voto para las elecciones del año 2000, que para las del año 1996. La complejidad de las condiciones políticas y sociales del contexto en que se desarrollaron las elecciones de 1996, pudieron afectar a dicho análisis, pues la economía no se presentaba como el factor fundamental de la discusión política, sino que la pérdida de reputación del Presidente socialista, por los escándalos asociados a su Gobierno, pudieron condicionar el estudio. Por ello considerábamos que las elecciones de 2011 suponían un escenario idóneo para volver a someter a verificación esta teoría. En este sentido, el análisis abordado pone de manifiesto un mayor peso de las variables económicas en el año 2000 que en el 2011. Así ocurre con las tres variables de contenido económico que se han medido en este trabajo, concluyendo un mayor premio al Gobierno por la situación de bonanza económica, que castigo en un contexto recesivo. Se ha 
contrastado, por tanto, la hipótesis de la asimetría del voto económico. De manera similar a los hallazgos de Fraile (2002), en este estudio también se concluye que las variables que dan cuenta del voto económico tienen una mayor importancia en la explicación del voto en los comicios que suceden en período de bonanza, que en los que se dan en una fase de recesión. En el caso de la investigación de Fraile (2002) se han apuntado diversas explicaciones basadas en factores contextuales muy específicos del caso de las elecciones generales de 1996, que podrían ayudar a profundizar en la explicación de este fenómeno. A las elecciones de 2011 el PSOE llegó con una importante crisis de reputación ${ }^{21}$, que se nutría no solo de su dificultad para gestionar la crisis económica, sino también de la pérdida de confianza para gestionar la política social, que había sido su principal bandera hasta entonces. Uno de los factores clave en esta pérdida de credibilidad en la dimensión social, vino determinado por la aprobación de una reforma exprés de la Constitución, incluyendo en el artículo 135 el concepto de estabilidad presupuestaria, lo que suponía anteponer el pago de la deuda del Estado a cualquier otro gasto, incluida la sanidad o la educación. A este respecto, también resulta necesario mencionar la presión que se ejerció desde las instituciones europeas, condicionando no sólo las políticas de ajuste a seguir, sino reforzando la idea de un gobierno debilitado y supeditado al interés económico, frente a una situación de alarma social, como se aprecia en el Gráfico 2. Igualmente, la pérdida de confianza en la figura del Presidente Zapatero y la percepción generalizada de que su mandado estaba agotado, dibujaban un escenario de cambio. A pesar de que la gestión económica había sido un elemento subyacente en dicha crisis de reputación desde las elecciones de 2008, es muy probable que en 2011 el factor económico se hubiera visto diluido por otras cuestiones, como los primeros indicios de una crisis de representación canalizada a través del movimiento $15 \mathrm{M}$ de ese año. Para los comicios analizados en este trabajo, se ha elaborado un modelo de voto económico que introduce las principales variables relacionadas con todos los debates teóricos abiertos en el ámbito de estudio. Este análisis profundiza en una línea de investigación en Ciencia Política que no hace sino enriquecerse, a medida que los contextos del comportamiento político ganan en complejidad y dinamismo.

\section{Bibliografía}

BALCELLS-VENTURA, L. (2003) «Elecciones y Accountability Económica de los Gobiernos: un Estudio Transnacional del voto al Incumbent en la Unión Europea». Paper presentado en el VI Congreso de la Asociación Española de Ciencia Política y de la Administración; Barcelona 18, 19 y 20 de septiembre de 2003.

21. Mientras en julio de 2004 , el 43,6\% de los entrevistados valoraban como buena o muy buena la gestión del gobierno socialista de José Luis Rodríguez Zapatero, apenas el 4,7\% lo valoraba en dichos términos en octubre de 2011. Fuente: Barómetros CIS. 
BELLUCCI, PLEWIS-BECK, M (2011) «A stable popularity function? Cross-national analysis». European Journal of Political Research 50(2), 190-211.

BLOOM, H y PRICE, D. (1975). «Voter Response to Short-Run Economic Conditions: the Asymmetric Effect of Prosperity and Recession». American Political Science Review, 1975, vol. 69, 04, 1240-1254. https://doi.org/10.2307/1955284

BOSCH, A., DÍAZ, A. y RIBA, C. (1999). «Las funciones de popularidad: Estado de la cuestión y principales debates». Reis, (85), 171-197. https://doi. org/10.2307/40184103

BOSCH, A. y RIBA, C. (2005). «Coyuntura económica y voto en España, 1985-1996». Papers, 75, 117-140. https://doi.org/10.5565/rev/papers/v75n0.1018

CAMPELL, A., CONVERSE, P., MILLAR, W. y STOKES, D. (1960). The American Voter. Nueva York: Wiley.

CONVERSE, P. (1964). The nature of belief systems in mass publics. En D. Apter, Ideology and Discontent. Nueva York: Free Pres.

DALTON, Russel J.; MCALLISTER, Ian y WATTENBERG, Martin P. (2002). The consecuencies of partisan dealignments. En Dalton, Russel J. y Wattenberg, Martin P. (ed.) (2002): Parties without Partisans. Political Change in Advanced Industrial Democracies. (pp. 37-63). Oxford: Oxford UP. https://doi.org/10.1093 /0199253099.003.0003

DOWNS, Anthony (1957). An Economic Theory of Democracy. New York: Harper.

DUVERGER, Maurice, 1957 [1951]. Los partidos políticos. México, fce.

FARRELL, David M. y WEBB, Paul (2002). Political parties as campaign organizations. En DALTON, RUSSEL J. y WATTENBERG, Martin P. (ed.) (2002). Parties without Partisans. Political Change in Advanced Industrial Democracies. (pp. 102-128). Oxford: Oxford UP.

FEARON JD. 1999. Electoral accountability and the control of politicians: selecting good types versus sanctioning poor performance. In Democracy, Accountability, and Representation, ed. A Przeworski, S Stokes, B Manin. New York: Cambridge Univ. Press

FESTINGER, L. (1957). A theory of cognitive dissonance. Evanston, Ill. [u.a.]: Row, Peterson.

FIORINA, M. (1981). Retrospective Voting in American nacional Elections. New Haven: Yale University Press.

FRAILE, M. (2002). «El voto económico en las elecciones de 1996 y 2000: una comparación». Revista Española De Ciencia Política, 6, 129-151.

FRAILE, M. (2005). Cuando la economía entra en las urnas El voto económico en España (1979-1996). Madrid: Centro de Investigaciones Sociológicas.

FRAILE, M. (2006). Cuánto saben los ciudadanos de política. Madrid: Fundación Alternativas.

GOODHART, C. y Bhansali, R. (1970). «Political Economy». Political Studies, 18(1), 43-106. https://doi.org/10.1111/j.1467-9248.1970.tb00659.x 
GONZÁLEZ, J. (1996). «Clases, ciudadanos y clases de ciudadanos. El ciclo electoral del pos-socialismo (1986-94)». Revista Española De Investigaciones Sociológicas, 74, 45-76. https://doi.org/10.2307/40183885

GRAMACHO, Wladimir (2006). Partidos Políticos versus Voto Económico. Un primer análisis comparado. Avance de Investigación. Universidad de Salamanca. GUNTHER, R y MONTERO, J. R (1998). «Los anclajes del partidismo: un análisis comparado del comportamiento electoral en cuatro democracias del sur de Europa», en P. del Castillo (ed.), Comportamiento político y electoral, Madrid: CIS, pp. 467-546.

HALL, P. (1993). El gobierno de la economía: implicaciones políticas de la intervención estatal en la economía en Gran Bretaña y Francia. Madrid: Ministerio de Trabajo y Seguridad Social.

KEY, V.O (1966). The Responsible Electorate: Rationality in Presidential Voting, 1936-1960. Cambridge: Harvard University Press. https://doi.org/10.4159/ harvard.9780674497764

KIEWIET, D. (1981). «Policy-Oriented Voting in Response to Economic Issues». The American Political Science Review, 75(2), 448-459. https://doi. org/10.2307/1961377

KINDER, D. y KIEWIET, D. (1981). «Sociotropic Politics: The American Case». British Journal Of Political Science, 11(02), 129-161. https://doi.org/10.1017/ S0007123400002544

KRAMER, G. (1971). «Short-Term Fluctuations in U.S. Voting Behavior, 18961964». American Political Sciencie Review, 65(01), 131-143. https://doi. org/10.2307/1955049

LAGO, I. y LAGO, S. (2011). «Descentralización y voto económico en España». Revista Española de Investigaciones Sociológicas, 136, 111-126. https://doi. org/10.5477/cis/reis.136.111

LAZARSFELD, P. F., BERELSON, B, and GAUDET, H. (1944) The People's Choice. New York: Duell, Sloan and Pearce.

LEWIS-BECK, M. (1988). Economics and elections. The Major Western Democracies. Ann Arbor: The University of Michigan Press.

LIPSET, S. y ROKKAN, S. (1967). Party systems and voter alignments: cross-national perspectives. New York: Free Press.

LORENTE FONTANEDA, Javier y SÁNCHEZ-VÍTORES, Irene (2018). "La desafección en las urnas: las elecciones generales de 2015 en España». Revista Española de Investigaciones Sociológicas, 161: 41-62.

MARAVALL, J. y PRZEWORSKI, A. (1999). "Reacciones políticas a la economía». Revista Española De Investigaciones Sociológicas, 87, 11-52. https://doi. org/10.2307/40184172

MARAVALL, J. y FRAILE, M. (2000). «Desempleo y política». Revista Española De Ciencia Política, 1(2), 7-42. 
MORA, A. (2017). El voto económico y estudio de agendas en España: aproximación a sus efectos en las elecciones generales del 2000 y 2011 (Tesis Doctoral). Universidad de Murcia, Murcia.

MUELLER, J. (1970). «Presidential Popularity from Truman to Johnson». American Political Science Review, 64(01), 18-34. https://doi.org/10.2307/1955610

NANNESTAD, P. y PALDAM, M. (1995). «It's the government's fault! A cross-section study of economic voting in Denmark, 1990/93». European Journal Of Political Research, 28(1), 33-62. https://doi.org/10.1111/j.1475-6765.1995.tb00486.x

NORRIS, Pippa (2000). The impact of televisión on civic malaise. En R. Putman y S. Pharr. Disaffected Democracies. Princeton: Princeton UP. https://doi. org/10.1515/9780691186849-014

O’DONNELL, G. (1998). «Accountability horizontal. «La Política: Revista de estudios sobre el estado y la sociedad, No 4, 1998, págs. 161-188 https://doi.org/10.22201/ fcpys.24484903e.1998.19.37205

POPKIN, S. (1991). The reasoning voter: Communication and Persuasion in Presidential Campaigns. Chicago: University of Chicago Press.

POWELL, G.B. \& WHITTEN, G. (1993). «A cross-national analysis of economic voting: Taking account of the political context». American Journal of Political Science 37(2): 391-414. https://doi.org/10.2307/2111378

RICO, G. (20002). «Candidatos y electores. la popularidad de los líderes políticos y su impacto en el comportamiento electoral». Revista de estudios políticos, № 124 , 2004, págs. 323-332.

SANDERS, D. (1996). «Economic performance, management competence and the outcome of the next general election». Political Studies, 44: 203-231. https://doi. org/10.1111/j.1467-9248.1996.tb00323.x

SANDERS, D., WARD, H., MARSH, D., \& FLETCHER, T. (1987). «Government Popularity and the Falklands War: A Reassessment». British Journal Of Political Science, 17(03), 281-313. https://doi.org/10.1017/S0007123400004762

SANDERS, D. (2000). «The Real Economy and Perceived Economy in Popularity Functions: How Much do Voters Need to Know? A Study of British Data 1974-97». Electoral Studies, 19: 275-294. https://doi.org/10.1016/S0261-3794(99)00052-9

SCHUMPETER, J. (1984) (1945). Capitalismo, socialismo y democracia. Barcelona: Ediciones Folio.

SHAPIRO, Robert Y. (1997). Reviewed Work: The New American Voter by Warren E. Miller, J. Merrill Shanks. Political Science Quarterly, 112(2), 313-314. https://doi. org/10.2307/2657948

SIMON, H. (1957). Models of Man, Social and Rational: Mathematical Essays on Rational Human Behavior in a Social Setting. Nueva York: Wiley 